Geopolítica(s) Revista de estudios sobre espacio y poder ISSN: 2172-3958

http://dx.doi.org/10.5209/GEOP.54220

\title{
Comentario. La visión de Galicia de Plácido Castro: la interrelación entre la (geo)política interna y la externa ${ }^{1}$
}

Juan M. Trillo Santamaría y Rubén C. Lois González²

Sumario. 1. Introducción. 2. Breve perfil biográfico del autor. 3. Líneas de pensamiento para comprender los textos seleccionados. 4. Hacia una comprensión global de la obra y pensamiento de Plácido Castro. Bibliografía.

Cómo citar: Trillo Santamaría, Juan M., y Lois González, Rubén C. (2016) “Comentario. La visión de Galicia de Plácido Castro: la interrelación entre la (geo)política interna y la externa". Geopolítica(s). Revista de estudios sobre espacio y poder, vol. 7, núm. 2, 309-317.

\section{Introducción}

En el centenario del nacimiento de Plácido Castro, autor gallego del que trataremos aquí, una publicación coordinada por X. Ríos (2002) buscaba rescatar del olvido la memoria de un autor desconocido no solo fuera de Galicia, sino también dentro, incluso entre los propios círculos galeguistas. Sin duda, Xulio Ríos, director del Instituto Galego de Ánalise e Documentación Internacional (IGADI), ha jugado un papel fundamental en la recuperación de la figura y obra de Plácido Castro, un finisterrano (era originario de Corcubión) de amplia trayectoria, cuya formación personal y política en Reino Unido fue fundamental para conformar su pensamiento político. Ya en $1997 \mathrm{X}$. Ríos publicó una pequeña biografía del personaje (64 páginas), que todavía hoy conforma el intento más acabado por explicar su trayectoria vital en una monografía. Trabajos posteriores, sobre los que nos apoyaremos en gran parte en nuestro comentario, han intentado ir llenando lagunas en la investigación sobre el autor. Así, en 2002 se suma al trabajo ya referido la publicación de una selección de textos periodísticos de Plácido Castro, prologado por el propio $X$. Ríos. Cuatro años más tarde, y como fruto de un congreso sobre la figura de Plácido Castro en 2005, sale a la luz una obra coral editada por la Xunta de Galicia en la que se repasan las distintas facetas del personaje (VV. AA., 2006). Ya en el siglo XXI se edita una nueva selección de textos, esta vez dedicados en exclusiva a uno de los grandes temas tratados por el autor: Irlanda (Castro, 2011). A todo ello,

1 (Nota de la redacción) Comentario sobre los textos de Plácido Castro: "Irlanda libre", "En torno a un título" y "El IX Congreso de Nacionalidades Europeas: el ingreso de Galicia".

2 Departamento de Xeografía, Universidade de Santiago de Compostela

Email: juan.m.trillo.s@gmail.com 
no debe dejarse de mencionar la labor desarrollada por la Fundación Plácido Castro desde su creación en 1999, que comentaremos más adelante.

El haber elegido la figura de Plácido Castro para esta sección de clásicos debe entenderse dentro de esta línea que persigue rescatar del olvido el pensamiento de un autor que, sin duda, puede ser de gran interés para los lectores de Geopolíti$c a(s)$. Entendemos que debido precisamente a su desconocimiento la catalogación de "clásico" pueda parecer en inicio excesiva. Sin embargo, en la línea de autores como el mencionado X. Ríos, sostenemos que la relevancia de sus ideas, con múltiples conexiones en la época actual, podrían llevar a catalogarlo como tal, al menos en el contexto del galeguismo. Con el fin de poder interpretar la selección de los tres artículos periodísticos seleccionados dentro de la obra de Plácido Castro, en primer lugar, presentaremos a grandes trazos la trayectoria vital del autor; a continuación, sintetizaremos su pensamiento en algunas ideas principales; por último, volveremos a comentar los trabajos realizados en torno a su obra y apuntaremos algunos de los retos que aún quedan abiertos para ahondar en su legado.

\section{Breve perfil biográfico del autor}

Plácido R. Castro del Río nace en Corcubión (A Coruña), localidad de la Costa da Morte, el 25 de enero de 1902. Hijo de una familia acomodada — su padre, Plácido Castro Rivas, empresario de minas, armador de buques y abastecimiento de carbón, llegó a ser diputado por A Coruña-, muy pronto abandona Galicia para marcharse al Reino Unido. A la edad temprana de seis años, según sus cronistas, será inscrito como interno en el colegio Scarborough, y acabará estudiando filología inglesa en la Universidad de Glasgow. Su formación personal abarcará campos diversos, tales como el teatro, la pintura, la poesía, el periodismo y la política. De todo ello dará cuenta en sus distintas colaboraciones en prensa, destacando sus crónicas en El Pueblo Gallego. Lo que se ha venido a denominar su primera etapa británica, que es la de su formación personal e intelectual, culmina en 1930, cuando Plácido Castro se traslada a vivir a Galicia. Será entonces cuando entre en contacto directo con los grandes nombres del pensamiento galeguista de la época y se incorpore activamente a la acción política.

La etapa de compromiso galeguista (1931-1936) conforma sus años más activos en la causa del nacionalismo gallego, que dejará de lado tras la inhabilitación que ha de sufrir en 1936 por sus ideas políticas. Los contactos que tuvo con A. Villar Ponte durante muchos veranos (Insúa, 2006) se convierten en constantes reuniones con personajes como Castelao, Otero Pedrayo, Vicente Risco, Bóveda, Suárez Picallo, del Riego y tantos otros, en unos años en los que se integrará en las Irmandades da Fala de A Coruña y participa en la Asamblea fundacional del Partigo Galeguista en diciembre de 1931 en Pontevedra ${ }^{3}$. De hecho, formará parte de su dirección y, como comentaremos más adelante, llegó a ser secretario de relaciones exteriores. Asimismo, participó muy activamente en la defensa del Estatuto para Galicia, y prefirió no presentarse a diputado en Cortes por el partido para seguir

Para conocer con detalle las distintas etapas y personajes fundamentales en el pensamiento galeguista sigue siendo de referencia indiscutible la obra de J. Beramendi (2007). 
desempeñando sus actividades a pie de calle en pro de la difusión de la necesidad de un modelo político que asegurase la defensa de los derechos de Galicia. Como ha señalado R. Villares (2006), Plácido Castro pertenece a la generación de los que nacen en los primeros años de siglo, comienzan a despuntar en la década de los 1920 y se incorporan de lleno a la vida pública en los tiempos de la II República. Generación que se verá truncada por la Guerra Civil. En el caso de Plácido Castro, será condenado a ocho años de destierro lejos de su casa, multa de 25.000 pesetas y 15 años de inhabilitación.

Esta condena supone un gran golpe para Plácido Castro, que abandona en gran medida sus actividades y escritos políticos. Desde entonces, aunque sin renegar de sus ideas, se dedicará más a otros temas ligados a asuntos culturales, sociales o económicos, o a su faceta de poeta o pintor. Tras una época en la que vive en Coruxo y Vigo (1936-1948), y una breve estancia en Lisboa, acaba retornando a Londres (1949-1955), donde vuelve a escribir crónicas para periódicos como La Nación de Buenos Aires o el Jornal de Noticias de Porto, y donde colabora como redactor-locutor en la BBC, en los programas de lengua gallega, española y portuguesa ${ }^{4}$.

En 1957 volverá a Galicia, y se incorpora como profesor de inglés en un instituto de Vilagarcía de Arousa. Escribirá en la prensa (Faro de Vigo, Diario de Pontevedra, La Noche, El Pueblo Gallego) y continuará su labor de traducción, una de sus facetas que ha despertado mayor interés en la crítica (Arias, 2006; Lorenzo, 2006; Araguas, 2002). No es de desdeñar que tradujese a autores como Rossetti, Yeaths o Hardy al gallego. Diez años después de su segundo retorno de Londres fallecerá el 17 de julio de 1967.

\section{Líneas de pensamiento para comprender los textos seleccionados}

Las múltiples caras de Plácido Castro, que fueron asomando en virtud de distintos tiempos y contextos de su vida, llevan a X. Ríos a referirse a él como "librepensador, dandi transterrado, dotado de múltiples cualidades dispersas (publicista, músico, escritor, erudito político, traductor, periodista, astrónomo, profesor de inglés...) [quien] tuvo una determinante actividad en la vida política y cultural de su tiempo" . En su etapa política más activa, en los años 1930, el autor se posicionaría en el espectro de la izquierda galeguista o, al menos, en una posición abiertamente liberal, democrática, antitotalitaria y progresista (Gómez, 2002). En todo caso, será un defensor del nacionalismo como el modelo social y político que puede garantizar el universalismo y la paz. Para Castro, solo desde la defensa y el reconocimiento de lo propio es posible acabar reconociendo la valía de los demás:

El verdadero patriotismo solo se siente cuando se ama lo esencial de la propia tierra $[\ldots]$ Los que sienten ese verdadero patriotismo serán los primeros en apre-

\footnotetext{
4 Sin duda, la emisión de programas en gallego desde la BBC londinense es un hecho a destacar en una época en la que la dictadura reprimía el uso de las lenguas distintas a la castellana en España. Una recopilación de los programas de la BBC puede consultarse en Toro (1994).

5 Así lo define en la presentación de la obra en la que se recoge una selección de artículos periodísticos de Plácido Castro (Castro, 2002: 5).
} 
ciar y respetar el patriotismo de los demás. De forma que el nacionalismo lejos de constituir un motivo de apartamiento y de división, viene a ser un agente de aproximación y de pacificación mucho más eficaz que las ideas internacionalistas, vanas e inútiles porque nadie las siente con convicción ${ }^{6}$.

La necesidad de respetar la idiosincrasia de cada nación y de asegurar su convivencia requiere de un sistema político que sea capaz de seguir estas premisas. Plácido Castro veía en el modelo británico un ejemplo a imitar. Consideraba que el Reino Unido era un país pequeño, pero poderoso en el mundo, que supo conjugar la identidad plural en su Imperio. Recordemos que en aquella época aún el Imperio británico seguía en pie, y que será con el Estatuto de Westminster de 1931 cuando se constituya la moderna Commonwealth (Pérez-Barreiro, 2006). Para Castro, el modelo británico sabía conjugar la existencia de distintas naciones en su seno, y no era extraño, por ejemplo, que un galés llegase a ser primer ministro británico, como fue el caso de Lloyd George. Irlanda sería, sin lugar a duda, la nación por la que mostrará más apego el autor, como tantos otros galeguistas de la época:

Un viaje a Irlanda tiene que ser para un gallego una peregrinación emocionante [...] Los lazos tradicionales y raciales hacen que esa isla sea para nosotros la nación más interesante del mundo ${ }^{7}$.

Plácido Castro viajará a Irlanda y la conocerá de primera mano (una verdadera excepción entre los galeguistas, vid. Ríos, 1997) en la época del Estado Libre Asociado. Fruto del Tratado anglo-irlandés de 1921, tras una guerra entre Gran Bretaña y el denominado Parlamento irlandés (Dáil Éiream), Irlanda alcanza unos niveles de autonomía amplios, aunque aún como dominio británico. No obstante, los primeros gobiernos de Cosgrave ahondarán en el reconocimiento internacional del nuevo país, tanto en la Sociedad de Naciones como en las Conferencias Imperiales. Este rol jugado por Irlanda en el desarrollo de lo que será la moderna Commonwealth a través de cambios graduales, el reconocimiento de la diferencia y los acuerdos entre socios es lo que admiraba Plácido Castro. Ahora bien, como acertadamente indica Pérez-Barreiro (2002, 2006), si en algún momento se enfrentaban los intereses de la Corona y los de Irlanda, Castro optará por la segunda. Y esto es lo que ocurrió en 1932 cuando el nuevo presidente De Valera reivindica mayor independencia, aunque sea recurriendo a un motivo de índole más simbólica que económica. He aquí el contexto para enmarcar el contenido del primer artículo seleccionado, "Irlanda Libre". A pesar de las ventajas que, para el autor, gozaba Irlanda en el Imperio, el país "arriesgó su bienestar" por un juramento de poco contenido práctico, pero sí simbólico. Y comparte esta actitud, porque "en el nacionalismo el sentimiento es el factor más potente".

Plácido Castro, al contrario que muchos otros galeguistas, no mantenía una relación de animadversión hacia Inglaterra. Como acabamos de ver, él no veía problemas en defender la realidad nacional de Irlanda en el contexto británico. Es por ello que reivindica la necesidad de no mantener posiciones beligerantes hacia In-

“El nacionalismo y la paz”, El Pueblo Gallego, 8 de mayo de 1927.

"Irlanda e Galicia”, Céltiga, núm. 91, 10 de octubre de 1928. 
glaterra ${ }^{8}$. Es más, el ejemplo británico es el que reclama para una España que no encuentra la manera de integrar las distintas naciones en un sistema político. De hecho, llega a afirmar en algún artículo que la situación de Galicia es la de una colonia, bajo el yugo no solo del centralismo del Estado, sino también de algo aún peor, el "caciquismo centralista gallego"9. Esto es, según Plácido Castro, todo lo contrario de lo que pasaba en el Reino Unido con el ejemplo comentado de Lloyd George. Para Galicia era preferible que no hubiera políticos gallegos en Madrid, porque su alto nivel de "desgalleguización" no provocaba sino el reforzamiento del centralismo. Comparando el caso gallego con el catalán y vasco, que contaban, según el autor, con mejores condiciones, afirma:

Galicia estará indefensa porque sus representantes en Cortes, pertenecientes a diversos partidos nacionales, ajenos a Galicia, no sabrán unirse para defenderla y expresar con unanimidad el criterio de nuestra tierra, como no han sabido hacerlo hasta ahora ${ }^{10}$.

En el contexto de la II República, Plácido Castro no opta por una "desmembración de España", sino por un modelo político que integre la pluralidad ibérica, lo que Castelao llamó Hespaña (Castelao, 1994 [1944]). Quizás uno de los puntos más interesantes en este autor es la conexión que establece entre política interior y exterior, tanto para Galicia o las otras naciones ibéricas, como para España. Alcanzar un modelo territorial que integrase a todos solucionaría no solo las tensiones internas, sino que sería también la base de un papel reforzado del Estado de cara al exterior. Porque según Castro, España solo tendría autoridad internacional una vez que solucionara los problemas autonómicos internos ${ }^{11}$. Para ello, debería conformarse en "naciones autónomas", lo que le otorgaría un porvenir más brillante que la defensa de un Estado unitario. De este modo, se permitiría que Cataluña y el País Vasco acercaran posiciones con las "tierras hermanas" de Francia, y Galicia cumpliera la "misión histórica" de aproximarse a los países de la lusofonía, Portugal y Brasil, así como a las "tierras celtas". Y es más, la propia España podría hermanarse con las naciones americanas de habla castellana, además de Filipinas, lo que le lleva a referirse a los dos modelos que consideraba más relevantes en la esfera internacional: podría conformarse así una verdadera Sociedad de Naciones de raíz ibérica, a la altura de los pueblos de habla inglesa.

La acción exterior de Galicia, título incluso de un artículo donde sintetiza sus ideas en torno a esta cuestión ${ }^{12}$, debería girar en torno a tres núcleos:

a) La relación con el País Vasco y Cataluña, algo en lo que participó personalmente a través de las reuniones de Galeuzca ${ }^{13}$. La única esperanza para

\footnotetext{
Véase, por ejemplo, su artículo "Irlanda, Inglaterra y el mundo", El Pueblo Gallego, 12 de febrero de 1927.

"Colonia", A Nosa Terra, 1 de diciembre de 1930.

"Urgencia de la Autonomía”, El Pueblo Gallego, 15 de mayo de 1932.

"Arredor do noso universalismo", A Nosa Terra, 25 de julio de 1932.

"La política exterior de Galicia”, El Pueblo Gallego, 4 de agosto de 1933.

Galeuzca o Galeusca es el nombre con el que se conocen los distintos acuerdos firmados por partidos nacionalistas gallegos, vascos y catalanes, que se iniciaron en 1923.
} 
el autor de forjar un verdadero patriotismo reside en la "liberación de estos pueblos y en la intensificación de sus características peculiares".

b) La relación con los pueblos de cultura portuguesa.

c) La relación con los pueblos de origen celta, como sería por antonomasia el caso de Irlanda. Plácido Castro abraza el ideario mítico del celtismo como raíz étnica y cultural de Galicia, elemento compartido por gran parte del nacionalismo gallego (García Álvarez, 2002). En este punto se inserta el segundo artículo escogido, "En torno a un título", publicado en la revista Céltiga ${ }^{14}$, en el que el autor expone sus consideraciones acerca de esta cultura en relación con Irlanda y Galicia.

La fuerza expresiva de Plácido Castro queda de manifiesto en esta larga cita, en la que sintetiza su pensamiento sobre la acción exterior de Galicia, que tiene que conservar su identidad:

Todo esto lo podía hacer Galicia siendo gallega. Inspirándose en ese nacionalismo que nuestros enemigos llaman separatismo, cuando es verdaderamente nuestro punto de contacto con el mundo. Galicia galleguista no solo tendrá una intensa vida propia, sino que se hallará ligada a un conjunto de pueblos ibéricos, a una comunidad de países galaico-lusitanos, a una hermandad de tierras célticas proyectando en cada uno su espíritu propio, recibiendo de cada uno el estímulo peculiar de su cultura- Es decir, que tendrá una verdadera y genuina política exterior. Pero sin ser gallega no pasará Galicia de ser un trozo de territorio del Estado español, sin alma propia, flamenquizada poco a poco, y poseyendo como aspiración máxima la de caciquear con más o menos eficacia en los Ministerios madrileños. He ahí el universalismo de nuestros adversarios ${ }^{15}$.

En su lectura del autor desde una visión contemporánea, X. Ríos (2006) quiere encontrar una conexión entre sus ideas y la política de acción exterior desarrollada por los distintos gobiernos gallegos de la democracia, lo que se ha venido a llamar en ciencia política paradiplomacia (Aldecoa, 2001). Así, la relación con Portugal sería la base de la actual Eurorregión Galicia-Norte de Portugal (cfr. Trillo \& Lois, 2011), y las conexiones con las tierras celtas sustentarían las estrategias transnacionales del Arco Atlántico (cfr. Trillo \& Paül, 2015).

De vuelta a la década de 1930, y siguiendo con la temática de la acción exterior de Galicia, no puede dejarse de mencionar la asistencia de Plácido Castro al IX Congreso de Nacionalidades Europeas celebrado en Berna (Suiza) ${ }^{16}$, los días 16, 17 y 19 de septiembre de 1933, de lo que da cuenta en el tercero de los textos seleccionados, publicado en El Pueblo Gallego. Plácido Castro acude en representación del Partido Galeguista, y defenderá una declaración en la que se afirmaba la condi-

14 Céltiga fue una importante revista de la inmigración gallega en Argentina, editada en Buenos Aires entre 1924 y 1932. Puede consultarse la integridad de sus números en URL: $<$ http://consellodacultura.gal/fondos_documentais/celtiga/>.

15 "La política exterior de Galicia”, El Pueblo Gallego, 4 de agosto de 1933.

16 El Congreso de Nacionalidades Europeas fue una organización internacional que reunió a representantes de minorías nacionales y formaciones políticas de naciones/nacionalidades sin Estado. Se constituyó en 1925 y desaparece en 1938. Para una información completa, véase Núñez Seixas (1992). 
ción de Galicia como nación, sustentada en una memoria presentada previamente y redactada por Vicente Risco ${ }^{17}$. Esta iniciativa parte de una visita a España en la primavera de 1933 del secretario general del Congreso de Nacionalidades Europeas, el periodista estonio Edwal Ammende, para conocer de cerca los casos vascos y catalán, que ya llevaban algunos años participando en el Congreso. Otero Pedrayo aprovechó la ocasión para darle a conocer el caso gallego (Ríos, 1992). De hecho, el propio Otero se anunció como representante gallego en el IX Congreso junto a Plácido Castro, pero acabó por acudir solo este.

Este acontecimiento es resaltado como un momento clave en el reconocimiento internacional de Galicia. Así, en el 70 aniversario de su celebración, la Fundación Plácido Castro con el apoyo siempre constante del IGADI y X. Ríos organizaron un homenaje que acabó en forma de libro (Ríos, 2004). Ya el propio Plácido Castro destacaba la importancia del reconocimiento adquirido en el Congreso de las Nacionalidades Europeas. Un Congreso, no obstante, al que no se volvería a acudir, y que estaba a punto de desaparecer unos años después, inmerso en las tensiones de una Europa que se encaminaba a la II Guerra Mundial. En un artículo publicado poco después del recogido en nuestra selección, el autor especifica los resultados de la consideración de Galicia como nacionalidad europea ${ }^{18}$. En primer lugar, Galicia queda "proclamada moralmente como una nación", lo que conlleva, en segundo punto, un fortalecimiento de la universalización de la misma, pues se integra en el conjunto de naciones mundiales. En tercer lugar, quedaría reforzado el acuerdo Galeuzka, ya que vascos y catalanes formaban parte previamente del Congreso. Y, por último, Galicia reafirma su apuesta por conformar una federación de naciones a la escala que se considere más pertinente: "Federación hispánica, ibérica, europea o mundial, con todas se declara compatible el nacionalismo gallego". Un año después, en la tercera Asamblea del Partigo Galeguista (Ourense, 1934), Plácido Castro sería elegido responsable de relaciones internacionales del partido.

\section{Hacia una comprensión global de la obra y pensamiento de Plácido Castro}

Como apuntábamos al inicio, la labor desarrollada en los últimos años tanto por el IGADI como por la Fundación Plácido Castro está siendo de gran relevancia para sacar del olvido a un personaje particular en la esfera del galeguismo ${ }^{19}$. De hecho, fue el IGADI el principal impulsor de la creación en 1999 de la Fundación, dirigida a reivindicar y divulgar la personalidad y pensamiento del autor. En la actualidad, su patronato incluye a tres municipios (Cambados, Corcubión y Vilagarcía de Arousa), la Asociación de Traductores Galegos y el propio IGADI. A través de su página web pueden consultarse materiales relacionados con el autor y seguir la actividad de la Fundación; entre sus actividades, cabe destacar la concesión del

17 Una exposición acerca del reconocimiento de Galicia como nación en el IX Congreso de las Nacionalidades Europeas, interpretada tanto desde el contexto internacional como el del galeguismo, puede encontrarse en Ríos (1992). Se recoge asimismo la memoria presentada a dicho congreso de la pluma de Vicente Risco.

18 "Galicia, nacionalidad europea", El Pueblo Gallego, 10 de octubre de 1933.

19 Pueden consultarse sendas páginas web: $<$ http://www.igadi.org/web/> y $<$ http://www.fundacionplacidocastro.com/ $>$. 
premio anual de traducción o la celebración de las conferencias anuales Plácido Castro.

Personaje particular, decíamos, sobre todo por su formación en el Reino Unido, y por su personal lectura de la realidad española y gallega a través de las lentes del modelo británico. De sus diversas facetas, la que más nos ha interesado aquí es la de cronista y político, que desarrolla con mayor intensidad desde finales de los años 1920 hasta 1936. Desgraciadamente, la guerra civil puso freno a la pluma de Plácido Castro en lo que se refiere a asuntos de marcada índole política. Así, por ejemplo, desconocemos su pensamiento posterior sobre la evolución histórica de Irlanda, o sus reflexiones en torno a la continuación del movimiento galeguista en tiempos de la dictadura de Franco.

En realidad, Plácido Castro no escribió ningún compendio de pensamiento político, sino que sus ideas se iban exponiendo en los artículos de prensa. Sus ensayos se dirigirían más hacia las cuestiones relacionadas con la traducción. Como argumentan algunos de los autores aquí reseñados, que se han dedicado a estudiar determinados aspectos de Plácido Castro, aún queda espacio por profundizar en su figura y pensamiento. Así, por ejemplo, un esfuerzo por recoger diversos materiales que pueden estar aún hoy dispersos podría dar lugar a una monografía que se introdujera con detenimiento en su vida personal, intelectual y política, y que nos narrara su historia desde una contextualización de su época.

Sea como fuere, nuestra pretensión aquí no ha sido otra que la de aportar un grano de arena más en la tarea de dar a conocer el pensamiento de Plácido Castro. Esperamos que la lectura de estas páginas inciten a acercarse a la obra de un autor que debería dejar de ser un desconocido para los lectores de Geopolítica(s).

\section{Bibliografía}

Aldecoa, F. (2001) Paradiplomacia. Las relaciones internacionales de las regiones. Madrid, Barcelona: Marcial Pons Ediciones Jurídicas y Sociales.

Araguas, V. (2002) "O traductor”, en X. Ríos (coord.) Plácido Castro no centenario. Baiona: IGADI, 89-100.

Arias, V. (2006) "Plácido Castro e a traducción ao galego", en VV. AA.: Congreso sobre Plácido Castro e o seu tempo. Santiago de Compostela: Xunta de Galicia, Consellería de Cultura e Deporte, 199-216.

Beramendi, J. (2007) De provincia a nación: historia do galeguismo político. Vigo: Xerais.

Castelao, A. (1994 [1944]) Sempre en Galiza. Madrid: Akal.

Castro, P. (2002) Unha escolma xornalística. Sada: Edicións do Castro.

Castro, P. (2011) Irlanda en Plácido Castro. Redondela: IGADI.

García Álvarez, J. (2002) Territorio y nacionalismo. La construcción geográfica de la identidad gallega. Santiago de Compostela: Xunta de Galicia.

Gómez, S. (2002) "El tiempo de Plácido Castro”, en X. Ríos (coord.) Plácido Castro no centenario. Baiona: IGADI, 105-126.

Insúa, E.X. (2006) “Antón Villar Ponte e Plácido Castro", en VV. AA.: Congreso sobre Plácido Castro e o seu tempo. Santiago de Compostela: Xunta de Galicia, Consellería de Cultura e Deporte, 33-75. 
Lorenzo, M. (2006) "Significado e valor da traducción de As Rubáiyát de Omar Khayyám", en VV. AA.: Congreso sobre Plácido Castro e o seu tempo. Santiago de Compostela: Xunta de Galicia, Consellería de Cultura e Deporte, 255-268.

Núñez Seixas, X. M. (1992) El problema de las nacionalidades en la Europa de entreguerras: el Congreso de Nacionalidades Europeas (1925-1938). Tesis Doctoral. European University Institute, Florencia.

Pérez-Barreiro, F. (2002) "Plácido Castro e o problema político de Irlanda", en X. Ríos (coord.) Plácido Castro no centenario. Baiona: IGADI, 25-46.

Pérez-Barreiro, F. (2006) "Plácido Castro e a política británica e irlandesa do seu tempo", en VV. AA.: Congreso sobre Plácido Castro e o seu tempo. Santiago de Compostela: Xunta de Galicia, Consellería de Cultura e Deporte, 77-94.

Ríos, X. (1992) Galicia e a sociedade das nacións. Vigo: Galaxia.

Ríos, X. (1997) Plácido Castro. Vigo: Ir Indo.

Ríos, X. (coord.) (2002) Plácido Castro no centenario. Baiona: IGADI

Ríos, X. (coord.) (2004) Galicia, un país no mundo. Sada: Edicións do Castro.

Ríos, X. (2006) "Plácido Castro e a proxección exterior de Galicia", en VV. AA.: Congreso sobre Plácido Castro e o seu tempo. Santiago de Compostela: Xunta de Galicia, Consellería de Cultura e Deporte, 23-32.

Trillo, J.M. \& Lois, R. (2011) "La frontera como motivo de atracción: una breve mirada a las relaciones Galicia-Região Norte”. Geopolítica(s). Revista de estudios sobre espacio y poder, vol. 2, núm. 1, 109-134.

Trillo, J.M. \& Paül, V. (2015) A cooperación transfronteiriza e territorial. Santiago de Compostela: Universidade de Santiago de Compostela.

Toro, A. (ed.) (1994) Galicia desde Londres. Oleiros: Ed. Tambre.

Villares, R. (2006) “A Xeración de Plácido Castro”, en VV. AA.: Congreso sobre Plácido Castro e o seu tempo. Santiago de Compostela: Xunta de Galicia, Consellería de Cultura e Deporte, 9-22.

VV. AA. (2006) Congreso sobre Plácido Castro e o seu tempo. Santiago de Compostela: Xunta de Galicia, Consellería de Cultura e Deporte. 